\title{
Impact of Health Literacy on Frailty among Community-Dwelling Seniors
}

\author{
Chi Hsien Huang $1,2,3,+\oplus$, Yu-Cheng Lai ${ }^{2,+}{ }^{\circledR}$, Yi Chen Lee ${ }^{4}$, Xiao Tong Teong ${ }^{5,6}$, \\ Masafumi Kuzuya ${ }^{1,7}$ and Kuang-Ming Kuo ${ }^{8, *(D)}$ \\ 1 Department of Community Healthcare and Geriatrics, Nagoya University Graduate School of Medicine, \\ Nagoya 4668550 Japan; evaairgigaa@gmail.com (C.H.H.); kuzuya@med.nagoya-u.ac.jp (M.K.) \\ 2 Department of Family Medicine, E-Da Hospital, I-Shou University, Kaohsiung City 82445, Taiwan; \\ ed107116@edah.org.tw \\ 3 School of Medicine for International Students, I-Shou University, Kaohsiung City 82445, Taiwan \\ 4 Department of Nutrition Therapy, E-DA Hospital, Kaohsiung City 82445, Taiwan; ed103549@edah.org.tw \\ 5 Adelaide Medical School, The University of Adelaide, Adelaide, South Australia 5005, Australia; \\ xiaotong.teong@adelaide.edu.au \\ 6 South Australian Health and Medical Research Institute (SAHMRI), Adelaide, \\ South Australia 5001, Australia \\ 7 Institutes of Innovation for Future Society, Nagoya University, Nagoya 4648601, Japan \\ 8 Department of Healthcare Administration, I-Shou University, Kaohsiung City 82245, Taiwan \\ * Correspondence: kuangmingkuo@gmail.com; Tel.: +886-7-6151100 (ext. 7622); Fax: +886-7-6155150 \\ + Both authors contributed equally to this work.
}

Received: 6 November 2018; Accepted: 23 November 2018; Published: 26 November 2018

\begin{abstract}
Health literacy has been reported to have effects on health behavior change and health-related outcomes, but few studies have explored the association between health literacy and frailty. The aim of our study is to investigate the relationships between health literacy and frailty among community-dwelling seniors. This cross-sectional study enrolled 603 community-dwelling older adults (307 women) in residential areas, with a mean age of $70.9 \pm 5.82$ years. Health literacy was assessed using the Mandarin version of the European Health Literacy Survey Questionnaire. Physical frailty was defined by Fried frailty phenotype. Logistic regression was carried out to determine potential risk factors of frailty. In the multivariate logistic regression model, physical activity (Odds Ratio [OR] 1.47, 95\% Confidence Interval [CI] 1.06-2.03) and health literacy (sufficient vs. excellent: OR $2.51,95 \%$ CI 1.32-4.77) were associated with prefrailty and frailty. In subgroup analysis, pre-frailty and frailty were also negatively associated with health literacy in individuals with 'insufficiently active' (inadequate vs. excellent: OR 5.44, 95\% CI 1.6-18.45) and 'sufficiently/highly active' physical activity levels (sufficient vs. excellent: OR 2.41, 95\% CI 1.07-5.42). Therefore, in these community-dwelling elderly adults, health literacy was associated with pre-frailty and frailty regardless of age, gender, socio-economic status, and education level.
\end{abstract}

Keywords: health literacy; frailty; elderly

\section{Introduction}

Frailty is a clinical geriatric syndrome in the elderly characterized by high vulnerability and low resilience [1] This geriatric giant is associated with an increased likelihood of worse health outcomes including falls, disability, hospitalization, in institutionalization and mortality [2-5]. Moreover, frailty has been demonstrated to be related to stress, life satisfaction and quality of life [6]. Owing to the complex domains of physical, psychological, socio-economic, and environmental factors linked to frailty, a comprehensive multi-disciplinary and multi-step intervention approach including nutritional 
support and physical exercise is recommended for treating frail older populations [1]. However, due to poor awareness of frailty itself and prevention strategies for the elderly, the initial step in frailty management is promoting knowledge and improving self-awareness $[7,8]$.

Recently, health literacy, defined as the ability to read, understand, evaluate, and use health information to make reasoned, health-related decisions [9], has been reported to be associated with health behavior and outcomes among adults with chronic diseases such as obesity, cardiovascular disease, and diabetes $[10,11]$. No studies have explored the association between health literacy and frailty, except for one cross-sectional study which did not control for several confounding factors including diseases, prescription drugs, protein intake and physical activity [12]. Considering that the current strength and quality of evidence is insufficient, more studies are needed to clarify these relationships. Thus, we designed the present study to determine whether health literacy is associated with frailty in community-dwelling older adults.

\section{Experimental Section}

\subsection{Study Design}

This cross-sectional study was conducted from February to October 2017 in Kaohsiung City in southern Taiwan. We recruited participants by sending invitation letters and posting handbills at nine community centers located in three residential areas characterized as $>3000,300-3000$, and $<300$ people per square kilometer for urban, suburban, and rural areas, respectively. Only individuals older than 65 years old who could provide informed consent were eligible for inclusion and further investigation by a trained nurse. Those who had impairments in the Barthel index of activities of daily living, were unable to perform a 5-m walk test, had active cancers or incurable diseases with an estimated life expectancy of 6 months or less, and those who could not complete an interview owing to severe hearing or visual impairment were excluded. The study protocol was approved by the Institutional Review Board of E-Da Hospital (EMRP-105-79).

\subsection{Measures}

Baseline characteristics were acquired through interviews using questionnaires. Age, gender, body mass index (BMI), education level, annual income (none, $\leq 8000$ USD, >8000 USD), medical history and medication lists were obtained. Daily protein and calorie intake were assessed using a face-to-face interview with food frequency questionnaire conducted by a trained nurse. The information was confirmed by 24-h dietary recall conducted by a dietician via a telephone interview. Physical activity was evaluated using the Taiwanese version of the International Physical Activity Questionnaire (IPAQ), and was further categorized into highly active, sufficiently active, and insufficiently active groups [13]. Geriatric depression scale-5 (GDS-5), a five-item questionnaire, was used to identify depression by a cut-off of 2 points [14].

Health literacy was assessed using the validated Mandarin version of the European Health Literacy Survey Questionnaire $[15,16]$. Three dimensions of health literacy including health care health literacy (HCHL), disease prevention health literacy (DPHL), and health promotion health literacy (HPHL) composed a 47-item self-reported questionnaire (HLS-EU-Q47) [17]. To facilitate clinical practice and enhance community awareness, a cross-validated 16-item short form (HLS-EU-Q16) has been developed [18]. We used the Mandarin version of the HLS-EU-Q16 and answers were coded as follows: 1 = very difficult, 2 = difficult, 3 = easy, and $4=$ very easy. To reduce ambiguous responses, the option 'don't know' was excluded from our version. The total sum score was generated and converted to a 0-50-point index scale. The level of health literacy was thus categorized into four groups of inadequate (score 0 to 25), problematic (score 26 to 33), sufficient (score 34 to 42), and excellent (score 43 to 50 points) health literacy groups [17].

In this study, frailty was defined as fulfilling three out of five phenotypic criteria: unintentional weight loss, fatigue, slow walking speed, low physical activity, and weakness consistent with those 
used in the Cardiovascular Health Study (CHS) [5]. Individuals meeting one or two criteria were defined as having a pre-frail status, and those who met none of the criteria were defined as being robust. A loss of $\geq 3$ kilograms or $\geq 5 \%$ of body weight in the past year was defined as unintentional weight loss [19]. Exhaustion was identified by two statements from the Center for Epidemiological Studies-Depression (CES-D) scale: (a) I felt that everything I did was an effort; (b) I could not get going [20]. The positive criterion is defined as the presence of at least one condition for three days or more during the last week [5]. Slow gait speed was defined as the lowest quintile in a 5-m walking speed test without acceleration and deceleration stratified by gender and height. In men, the cutoff points of those whose heights were $\leq 168$ and $>168 \mathrm{~cm}$ were 0.89 and $0.96 \mathrm{~m} / \mathrm{s}$, respectively; while corresponding values in women whose heights were $\leq 156$ and $>156 \mathrm{~cm}$ were 0.85 and $0.88 \mathrm{~m} / \mathrm{s}$, respectively. Low physical activity was defined as the lowest quintile of activity level assessed using the Taiwanese version of the IPAQ [13]. The following cutoff values stratified by gender were used: $\leq 1236 \mathrm{kcal} /$ week for men, $\leq 1212 \mathrm{kcal} /$ week for women. Weakness was defined by low handgrip strength as measured by a digital dynamometer (TTM-YD, Tokyo, Japan). The cut-off values of handgrip strength across increasing quintiles stratified by gender and BMI $(\leq 22.6,22.61-24.8$, $24.81-26.7,>26.7 \mathrm{~kg} / \mathrm{m}^{2}$ ) were $20.9,21.6,22$, and $22.5 \mathrm{~kg}$ in men, and $13,15.4,16.8$, and $16.4 \mathrm{~kg}$ in women, respectively.

\subsection{Statistical Analysis}

We analyzed data using IBM SPSS Statistics for Windows, Version 25.0. (IBM Corp., Armonk, NY, UK). First, demographic data including number, percentage, mean and standard deviation were summarized by gender. Comparisons between men and women were based on an analysis of variance (for continuous variables) and on a chi-square test (for dichotomous variables). A $p$-value of 0.05 was used to determine statistical significance. A logistic regression model was used to determine the associations between health literacy and prefrailty and frailty. Participant characteristics and potential variables were entered in a simultaneous model with adjustments.

\section{Results}

\subsection{Characteristics of the Participants}

The mean age of all respondents $(n=603)$ was $70.9 \pm 5.82$ years, with men being on average older than the women (Table 1). There were significant differences in the level of education and health literacy between male and female participants. Although more of the men $(36.15 \%)$ had an education level above junior high school than the women $(22.74 \%)$, slightly more women had sufficient and excellent health literacy (44.62\%) compared to men (42.92\%) (Figure 1). Total daily calorie and protein intake were $20.25 \pm 4.97 \mathrm{kcal} / \mathrm{kg} /$ day and $0.66 \pm 0.19 \mathrm{~g} / \mathrm{kg} /$ day, respectively (Table 1$)$. With respect to physical activity and anthropometric measures, men were more physically active, had a faster walking speed and greater handgrip strength than women (Table 1). With regards to frailty, $4.05 \%$ and $19.32 \%$ of the men had pre-frailty and frailty respectively, compared to $1.95 \%$ and $54.72 \%$ of women. Additional descriptive statistics are shown in Table 1.

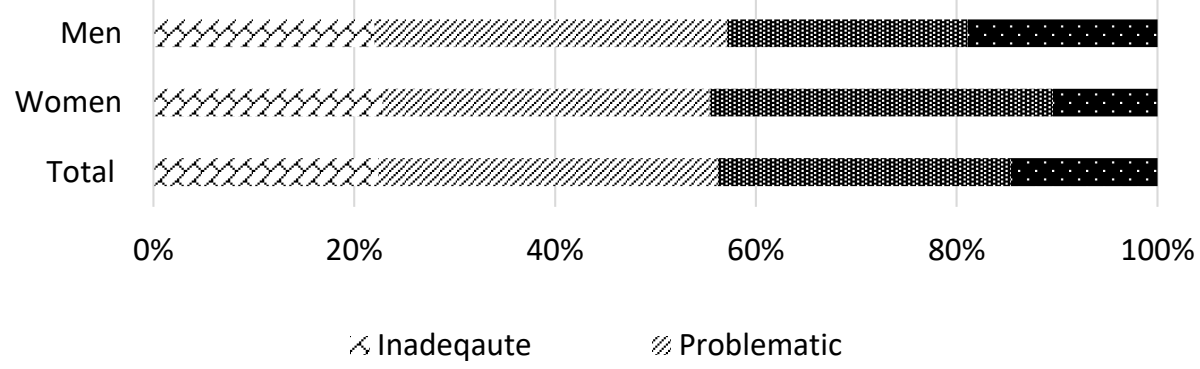

Figure 1. Level of health literacy by sex. 
On the other hand, participants with better health literacy were younger and highly educated and had higher income, fewer morbidities, less polypharmacy, and less depressive moods. Moreover, they were more physically active, with faster walking speed and greater hand grip strength. Detailed descriptive profiles are shown in Table 2.

Table 1. Characteristics of the participants by sex

\begin{tabular}{|c|c|c|c|c|}
\hline Characteristics & Total $(n=603)$ & Men $(n=296)$ & Women $(n=307)$ & $p$ Value * \\
\hline Age, years (mean \pm standard deviation [SD]) & $70.9 \pm 5.82$ & $72.04 \pm 6.45$ & $69.81 \pm 4.90$ & $<0.001$ \\
\hline BMI, $\mathrm{kg} / \mathrm{m}^{2}($ mean $\pm \mathrm{SD})$ & $24.67 \pm 2.99$ & $24.52 \pm 2.84$ & $69.81 \pm 4.10$ & 0.23 \\
\hline \multicolumn{5}{|l|}{ Education level, $n(\%)$} \\
\hline Illiterate & $128(21.23 \%)$ & $43(14.53 \%)$ & $85(27.69 \%)$ & \multirow[t]{4}{*}{$<0.001$} \\
\hline Elementary school & $299(49.59 \%)$ & $146(49.32 \%)$ & $153(49.84 \%)$ & \\
\hline Junior and senior high school & $130(21.56 \%)$ & $70(23.65 \%)$ & $60(19.54 \%)$ & \\
\hline College or above & $46(7.63 \%)$ & $37(12.5 \%)$ & $9(2.93 \%)$ & \\
\hline \multicolumn{5}{|l|}{ Health literacy, $n(\%)$} \\
\hline Inadequate & $135(22.4 \%)$ & $65(22.0 \%)$ & $70(22.8 \%)$ & \multirow[t]{4}{*}{0.004} \\
\hline Problematic & $204(33.83 \%)$ & $104(35.14 \%)$ & $100(32.57 \%)$ & \\
\hline Sufficient & $176(29.19 \%)$ & $71(24 \%)$ & $105(34.2 \%)$ & \\
\hline Excellent & $88(14.59 \%)$ & $56(18.92 \%)$ & $32(10.42 \%)$ & \\
\hline \multicolumn{5}{|l|}{ Annual income, $n(\%)$} \\
\hline No income & $46(7.63 \%)$ & $17(5.74 \%)$ & $29(9.45 \%)$ & \multirow[t]{3}{*}{0.08} \\
\hline$\leq 8000$ USD & $389(64.51 \%)$ & $187(63.18 \%)$ & $202(65.8 \%)$ & \\
\hline$>8000$ USD & $168(27.86 \%)$ & $92(31.08 \%)$ & $76(24.75 \%)$ & \\
\hline \multicolumn{5}{|l|}{ Multiple comorbidities, No. of diseases (\%) } \\
\hline $0-2$ & $521(86.4 \%)$ & $257(86.82 \%)$ & $264(86 \%)$ & \multirow[t]{2}{*}{0.77} \\
\hline$\geq 3$ & $82(13.6 \%))$ & $39(13.18 \%)$ & $43(14 \%)$ & \\
\hline \multicolumn{5}{|l|}{ Polypharmacy, No. of medications (\%) } \\
\hline$<5$ & $183(30.35 \%)$ & $93(31.42 \%)$ & $90(29.32 \%)$ & \multirow[t]{2}{*}{0.57} \\
\hline$\geq 5$ & $420(69.65 \%)$ & $203(68.58 \%)$ & $217(70.68 \%)$ & \\
\hline \multicolumn{5}{|l|}{ GDS-5 score } \\
\hline$<2$ & $483(80.1 \%)$ & $238(80.41 \%)$ & $245(79.8 \%)$ & \multirow[t]{2}{*}{0.85} \\
\hline$\geq 2$ & $120(19.9 \%)$ & $58(19.59 \%)$ & $62(20.2 \%)$ & \\
\hline \multicolumn{5}{|l|}{ Physical activity (IPAQ), $n$ (\%) } \\
\hline Insufficiently active & $281(46.6 \%)$ & $132(44.59 \%)$ & $149(48.53 \%)$ & \multirow[t]{3}{*}{0.02} \\
\hline Sufficiently active & $284(47.1 \%)$ & $137(46.28 \%)$ & $147(47.88 \%)$ & \\
\hline Highly active & $38(6.3 \%)$ & $27(9.12 \%)$ & $11(3.58 \%)$ & \\
\hline $\begin{array}{c}\text { Calorie intake, } \mathrm{kcal} / \mathrm{kg} / \text { day }(\text { mean } \pm \mathrm{SD}) \\
\text { Macronutrients }\end{array}$ & \multicolumn{3}{|c|}{ Macronutrients } & 0.18 \\
\hline Carbohydrate intake, $\mathrm{g} / \mathrm{kg} /$ day & $3.01 \pm 0.85$ & $2.97 \pm 0.78$ & $3.06 \pm 0.92$ & 0.17 \\
\hline Fat intake, $\mathrm{g} / \mathrm{kg} /$ day & $0.61 \pm 0.2$ & $0.61 \pm 0.18$ & $0.62 \pm 0.21$ & 0.3 \\
\hline Protein intake, $\mathrm{g} / \mathrm{kg} /$ day & $0.66 \pm 0.19$ & $0.66 \pm 0.17$ & $0.67 \pm 0.20$ & 0.87 \\
\hline Walking speed, $\mathrm{m} / \mathrm{s}$ (mean $\pm \mathrm{SD})$ & $1 \pm 0.2$ & $1.03 \pm 0.17$ & $0.97 \pm 0.22$ & $<0.001$ \\
\hline Handgrip, $\mathrm{kg}$ (mean \pm SD) & $23.46 \pm 7.74$ & $28.14 \pm 7.45$ & $18.95 \pm 4.75$ & $<0.001$ \\
\hline \multicolumn{5}{|l|}{ Frailty status, $n(\%)$} \\
\hline Robust & $271(44.94 \%)$ & $138(46.62 \%)$ & $133(43.32 \%)$ & \multirow[t]{3}{*}{0.18} \\
\hline Pre-frailty & $314(52.07 \%)$ & $146(49.32 \%)$ & $168(54.72 \%)$ & \\
\hline Frailty & $18(2.99 \%)$ & $12(4.05 \%)$ & $6(1.95 \%)$ & \\
\hline
\end{tabular}

GDS-5, Geriatric depression scale-5; IPAQ, International Physical Activity Questionnaires. ${ }^{*}$ Comparison of mean value of the characteristics between men and women.

Table 2. Characteristics of the participants by health literacy (HL).

\begin{tabular}{|c|c|c|c|c|c|}
\hline Characteristics & $\begin{array}{c}\text { Inadequate } \mathrm{HL} \\
\quad(n=135)\end{array}$ & $\begin{array}{l}\text { Problematic } \\
\text { HL }(n=204)\end{array}$ & $\begin{array}{l}\text { Sufficient HL } \\
\quad(n=176)\end{array}$ & $\begin{array}{l}\text { Excellent HL } \\
\quad(n=88)\end{array}$ & $p$ Value * \\
\hline Age, years (mean \pm SD) & $73.2 \pm 6.1$ & $71.1 \pm 6.1$ & $70.0 \pm 4.9$ & $68.5 \pm 5.0$ & $<0.001$ \\
\hline $\mathrm{BMI}, \mathrm{kg} / \mathrm{m}^{2}($ mean $\pm \mathrm{SD})$ & $24.6 \pm 3.1$ & $24.7 \pm 3.2$ & $24.8 \pm 2.9$ & $24.4 \pm 2.4$ & 0.71 \\
\hline \multicolumn{6}{|l|}{ Education level, $n(\%)$} \\
\hline Illiterate & $56(41.5 \%)$ & $46(22.5 \%)$ & $23(13.1 \%)$ & $3(3.4 \%)$ & $<0.001$ \\
\hline Elementary school & $68(50.4 \%)$ & $126(61.8 \%)$ & $86(48.9 \%)$ & $19(21.6 \%)$ & \\
\hline Junior and senior high school & $11(8.1 \%)$ & $30(14.7 \%)$ & $63(35.8 \%)$ & $26(29.5 \%)$ & \\
\hline College-or-above & 0 & $2(1.0 \%)$ & $4(2.3 \%)$ & $40(45.5 \%)$ & \\
\hline \multicolumn{6}{|l|}{ Annual income, $n(\%)$} \\
\hline No income & $3(2.2 \%)$ & $14(6.9 \%)$ & $28(15.9 \%)$ & $1(1.1 \%)$ & $<0.001$ \\
\hline$\leq 8000$ USD & $83(61.5 \%)$ & $152(74.5 \%)$ & $110(62.5 \%)$ & $44(50.0 \%)$ & \\
\hline$>8000$ USD & $49(36.3 \%)$ & $38(18.6 \%)$ & $38(21.6 \%)$ & $43(48.9 \%)$ & \\
\hline \multicolumn{6}{|l|}{$\begin{array}{c}\text { Multiple comorbidities, No. of } \\
\text { diseases }(\%)\end{array}$} \\
\hline $0-2$ & $99(73.3 \%)$ & $178(87.3 \%)$ & $159(90.3 \%)$ & $85(96.6 \%)$ & $<0.001$ \\
\hline$\geq 3$ & $36(26.7 \%)$ & $26(12.7 \%)$ & $17(9.7 \%)$ & $3(3.4 \%)$ & \\
\hline
\end{tabular}


Table 2. Cont.

\begin{tabular}{|c|c|c|c|c|c|}
\hline \multicolumn{6}{|l|}{ Polypharmacy, No. of medication (\%) } \\
\hline$<5$ & $21(15.6 \%)$ & $58(28.4 \%)$ & $60(34.1 \%)$ & $44(50.0 \%)$ & $<0.001$ \\
\hline$\geq 5$ & $114(84.4 \%)$ & $146(71.6 \%)$ & $116(65.9 \%)$ & $44(50.0 \%)$ & \\
\hline \multicolumn{6}{|l|}{ GDS- $\overline{5}$ scores } \\
\hline$<2$ & $80(59.3 \%)$ & $165(80.9 \%)$ & $156(88.6 \%)$ & $82(93.2 \%)$ & $<0.001$ \\
\hline$\geq 2$ & $55(40.7 \%)$ & $39(19.1 \%)$ & $20(11.4 \%)$ & $6(6.8 \%)$ & \\
\hline \multicolumn{6}{|l|}{ Physical activity (IPAQ), $n$ (\%) } \\
\hline Insufficiently active & $94(69.9 \%)$ & $88(43.1 \%)$ & $61(34.1 \%)$ & $38(43.2 \%)$ & $<0.001$ \\
\hline Sufficiently active & $38(28.1 \%)$ & $100(49.0 \%)$ & $101(57.4 \%)$ & $45(51.1 \%)$ & \\
\hline Highly active & $3(2.2 \%)$ & $16(7.8 \%)$ & $14(8.0 \%)$ & $5(5.7 \%)$ & \\
\hline Calorie intake, $\mathrm{kcal} / \mathrm{kg} /$ day $($ mean $\pm \mathrm{SD})$ & $20.4 \pm 4.6$ & $20.4 \pm 5.6$ & $19.8 \pm 4.9$ & $20.4 \pm 4.2$ & 0.62 \\
\hline \multicolumn{6}{|l|}{ Macronutrients } \\
\hline Carbohydrate intake, $\mathrm{g} / \mathrm{kg} /$ day & $3.0 \pm 0.7$ & $3.1 \pm 1.0$ & $3.0 \pm 0.8$ & $3.0 \pm 0.8$ & 0.56 \\
\hline Fat intake, $\mathrm{g} / \mathrm{kg} /$ day & $0.6 \pm 0.2$ & $0.6 \pm 0.2$ & $0.6 \pm 0.2$ & $0.6 \pm 0.2$ & 0.17 \\
\hline Protein intake, $\mathrm{g} / \mathrm{kg} /$ day & $0.7 \pm 0.2$ & $0.7 \pm 0.2$ & $0.6 \pm 0.2$ & $0.7 \pm 0.2$ & 0.23 \\
\hline Walking speed, $\mathrm{m} / \mathrm{s}$ (mean $\pm \mathrm{SD})$ & $1.0 \pm 0.2$ & $1.0 \pm 0.2$ & $1.0 \pm 0.2$ & $1.1 \pm 0.2$ & $<0.001$ \\
\hline Handgrip, $\mathrm{kg}($ mean \pm SD) & $23.4 \pm 6.5$ & $23.3 \pm 7.3$ & $21.6 \pm 8.4$ & $27.8 \pm 7.5$ & $<0.001$ \\
\hline \multicolumn{6}{|l|}{ Frailty status, $n(\%)$} \\
\hline Robust & $46(34.1 \%)$ & $105(51.5 \%)$ & $64(36.4 \%)$ & $56(63.6 \%)$ & $<0.001$ \\
\hline Pre-frailty & $84(62.6 \%)$ & $93(45.6 \%)$ & $106(60.2 \%)$ & $31(35.2 \%)$ & \\
\hline Frailty & $5(3.7 \%)$ & $6(2.9 \%)$ & $6(3.4 \%)$ & $1(1.1 \%)$ & \\
\hline
\end{tabular}

GDS-5, Geriatric depression scale-5; IPAQ, International Physical Activity Questionnaires. * Comparison of mean value of the characteristics between participants with different health literacy.

\subsection{Relationships among Health Literacy, Pre-Frailty, and Frailty}

The univariate logistic regression model showed that education, health literacy, multiple comorbidities, GDS-5 scores, and physical activity were significantly associated with pre-frailty and frailty (Table 3). In the multivariate logistic regression model, physical activity (OR 1.47, 95\% CI 1.06-2.03) and health literacy (sufficient vs. excellent: OR 2.51, 95\% CI 1.32-4.77) remained significantly associated with prefrailty and frailty after adjusting for age, sex, BMI, education level, annual income, multiple comorbidities, polypharmacy, depression, and protein intake (Table 3). Individuals with 'insufficiently active' physical activity levels were at a higher risk of having pre-frailty and frailty (OR $1.55,95 \%$ CI 1.07-2.23) compared to those with 'sufficiently/highly active' physical activity (Table 3).

In subgroup analysis by physical activity, pre-frailty and frailty were negatively associated with annual income (0 vs. $>8000$ USD: OR 5.44, 95\% CI 1.01-29.17) and health literacy (sufficient vs. excellent: OR 4.12, 95\% CI 1.28-13.22; inadequate vs. excellent: OR 5.44, 95\% CI 1.6-18.45) in individuals with 'insufficiently active' physical activity (Table 4). On the other hand, for individuals with 'sufficiently/highly active' physical activity, sufficient health literacy was negatively correlated with pre-frailty and frailty (OR 2.41, 95\% CI 1.07-5.42) (Table 4). A high BMI was associated with a higher risk of having pre-frailty and frailty (OR 1.11, 95\% CI 1.02-1.21) in those with sufficiently/highly active physically active (Table 4).

Table 3. Risk factors for pre-frail and frail status by odds ratio in logistic regression analysis

\begin{tabular}{|c|c|c|c|c|c|c|c|c|}
\hline \multirow{4}{*}{ Variable } & \multicolumn{8}{|c|}{ Pre-Frailty and Frailty (Versus Robust) } \\
\hline & \multicolumn{4}{|c|}{ Univariate Model } & \multicolumn{4}{|c|}{ Multivariate Model * } \\
\hline & \multirow{2}{*}{$\begin{array}{l}\text { Odds } \\
\text { Ratio }\end{array}$} & \multicolumn{2}{|c|}{$95 \% \mathrm{CI}$} & \multirow{2}{*}{$p$ Value } & \multirow{2}{*}{$\begin{array}{l}\text { Odds } \\
\text { Ratio }\end{array}$} & \multicolumn{2}{|c|}{$95 \%$ CI } & \multirow{2}{*}{$p$ Value } \\
\hline & & $\begin{array}{l}\text { Lower } \\
\text { Limit }\end{array}$ & $\begin{array}{l}\text { Upper } \\
\text { Limit }\end{array}$ & & & $\begin{array}{l}\text { Lower } \\
\text { Limit }\end{array}$ & $\begin{array}{l}\text { Upper } \\
\text { Limit }\end{array}$ & \\
\hline $\begin{array}{c}\text { Age (years) } \\
\text { Sex }\end{array}$ & 1.03 & 1.00 & 1.06 & 0.07 & 1.02 & 0.98 & 1.05 & 0.33 \\
\hline Female & 1.00 & & & & 1.00 & & & \\
\hline Male & 0.88 & 0.63 & 1.21 & 0.42 & 1.06 & 0.74 & 1.52 & 0.76 \\
\hline $\mathrm{BMI}\left(\mathrm{kg} / \mathrm{m}^{2}\right)$ & 1.05 & 0.99 & 1.11 & 0.09 & 1.04 & 0.98 & 1.11 & 0.18 \\
\hline \multicolumn{9}{|l|}{ Education level } \\
\hline Illiterate & 2.84 & 1.42 & 5.71 & $<0.01$ & 1.66 & 0.65 & 4.24 & 0.29 \\
\hline Elementary school & 2.34 & 1.23 & 4.45 & 0.01 & 1.46 & 0.63 & 3.41 & 0.38 \\
\hline Junior/senior high school & 1.56 & 0.78 & 3.10 & 0.21 & 0.95 & 0.42 & 2.18 & 0.91 \\
\hline College or above & 1.00 & & & & 1.00 & & & \\
\hline \multicolumn{9}{|l|}{ Health literacy } \\
\hline Inadequate & 3.39 & 1.93 & 5.94 & $<0.01$ & 1.71 & 0.82 & 3.56 & 0.15 \\
\hline
\end{tabular}


Table 3. Cont.

\begin{tabular}{|c|c|c|c|c|c|c|c|c|}
\hline Problematic & 1.65 & 0.99 & 2.76 & 0.06 & 1.11 & 0.58 & 2.14 & 0.74 \\
\hline Sufficient & 3.06 & 1.80 & 5.21 & $<0.01$ & 2.51 & 1.32 & 4.77 & 0.01 \\
\hline Excellent & 1.00 & & & & 1.00 & & & \\
\hline \multicolumn{9}{|l|}{ Annual income } \\
\hline No income & 1.92 & 0.97 & 3.82 & 0.06 & 1.52 & 0.72 & 3.22 & 0.28 \\
\hline$\leq 8000$ USD & 1.14 & 0.79 & 1.64 & 0.48 & 0.99 & 0.65 & 1.51 & 0.96 \\
\hline$>8000$ USD & 1.00 & & & & 1.00 & & & \\
\hline \multicolumn{9}{|l|}{$\begin{array}{l}\text { Comorbidities } \\
\text { (No. of diseases) }\end{array}$} \\
\hline $0-2$ & 1.00 & & & & 1.00 & & & \\
\hline$\geq 3$ & 1.91 & 1.16 & 3.14 & 0.01 & 1.35 & 0.78 & 2.32 & 0.28 \\
\hline \multicolumn{9}{|l|}{$\begin{array}{c}\text { Polypharmacy } \\
\text { (No. of medications) }\end{array}$} \\
\hline$<5$ & 1.00 & & & & 1.00 & & & \\
\hline$\geq 5$ & 1.40 & 0.99 & 1.99 & 0.06 & 1.02 & 0.69 & 1.52 & 0.92 \\
\hline \multicolumn{9}{|l|}{ GDS-5 score } \\
\hline$<2$ & 1.00 & & & & 1.00 & & & \\
\hline$\geq 2$ & 1.92 & 1.26 & 2.93 & $<0.01$ & 1.58 & 0.99 & 2.52 & 0.053 \\
\hline \multicolumn{9}{|l|}{ Physical activity (IPAQ) } \\
\hline Insufficiently active & 1.47 & 1.06 & 2.03 & 0.02 & 1.55 & 1.07 & 2.23 & 0.02 \\
\hline Sufficiently/highly active & 1.00 & & & & 1.00 & & & \\
\hline \multicolumn{9}{|l|}{ Protein intake (g/kg/day) } \\
\hline$<0.8$ & 1.15 & 0.52 & 2.55 & 0.72 & 1.06 & 0.45 & 2.53 & 0.89 \\
\hline $0.8-1$ & 0.71 & 0.30 & 1.68 & 0.44 & 0.70 & 0.28 & 1.73 & 0.44 \\
\hline$\geq 1$ & 1.00 & & & & 1.00 & & & \\
\hline
\end{tabular}

GDS-5, Geriatric depression scale-5; IPAQ, International Physical Activity Questionnaires. * Adjusted R squared = 0.115.

Table 4. Adjusted odds ratios for pre-frail and frail status by physical activity

\begin{tabular}{|c|c|c|c|c|c|c|c|c|}
\hline \multirow{4}{*}{ Variable } & \multicolumn{8}{|c|}{ Pre-Frailty and Frailty (Versus Robust) } \\
\hline & \multicolumn{4}{|c|}{ Insufficiently Physically Active Category \# } & \multicolumn{4}{|c|}{$\begin{array}{c}\text { Sufficiently/Highly Physically Active } \\
\text { Category * }\end{array}$} \\
\hline & \multirow{2}{*}{$\begin{array}{l}\text { Odds } \\
\text { Ratio }\end{array}$} & \multicolumn{2}{|c|}{$95 \% \mathrm{CI}$} & \multirow{2}{*}{$p$ Value } & \multirow{2}{*}{$\begin{array}{l}\text { Odds } \\
\text { Ratio }\end{array}$} & \multicolumn{2}{|c|}{$95 \%$ CI } & \multirow[b]{2}{*}{$p$ Value } \\
\hline & & $\begin{array}{l}\text { Lower } \\
\text { Limit }\end{array}$ & $\begin{array}{l}\text { Upper } \\
\text { Limit }\end{array}$ & & & $\begin{array}{l}\text { Lower } \\
\text { Limit }\end{array}$ & $\begin{array}{l}\text { Upper } \\
\text { Limit }\end{array}$ & \\
\hline $\begin{array}{l}\text { Age (years) } \\
\text { Sex }\end{array}$ & \multicolumn{7}{|c|}{ Sex } & 0.09 \\
\hline Female & 1.00 & & & & 1.00 & & & \\
\hline Male & 1.51 & 0.87 & 2.65 & 0.15 & 0.75 & 0.44 & 1.26 & 0.28 \\
\hline Body weight index $[\mathrm{BMI}]\left(\mathrm{kg} / \mathrm{m}^{2}\right)$ & 1.02 & 0.92 & 1.13 & 0.68 & 1.11 & 1.02 & 1.21 & 0.02 \\
\hline \multicolumn{9}{|l|}{ Education level } \\
\hline Illiterate & 1.05 & 0.22 & 4.99 & 0.95 & 1.67 & 0.49 & 5.73 & 0.42 \\
\hline Elementary school & 1.90 & 0.45 & 7.95 & 0.38 & 0.79 & 0.26 & 2.39 & 0.67 \\
\hline Junior/senior high school & 1.13 & 0.29 & 4.49 & 0.86 & 0.62 & 0.21 & 1.86 & 0.39 \\
\hline College or above & 1.00 & & & & 1.00 & & & \\
\hline \multicolumn{9}{|l|}{ Health literacy } \\
\hline Inadequate & 5.44 & 1.60 & 18.45 & 0.01 & 0.56 & 0.19 & 1.69 & 0.31 \\
\hline Problematic & 2.62 & 0.81 & 8.51 & 0.11 & 0.73 & 0.32 & 1.68 & 0.46 \\
\hline Sufficient & 4.12 & 1.28 & 13.22 & 0.02 & 2.41 & 1.07 & 5.42 & 0.03 \\
\hline Excellent & 1.00 & & & & 1.00 & & & \\
\hline \multicolumn{9}{|l|}{ Annual income } \\
\hline No income & 5.44 & 1.01 & 29.17 & 0.048 & 0.98 & 0.37 & 2.57 & 0.96 \\
\hline$\leq 8000$ USD & 0.98 & 0.54 & 1.81 & 0.96 & 1.00 & 0.52 & 1.94 & 0.99 \\
\hline$>8000$ USD & 1.00 & & & & 1.00 & & & \\
\hline \multicolumn{9}{|l|}{$\begin{array}{l}\text { Multiple comorbidities } \\
\text { (No. of diseases) }\end{array}$} \\
\hline $0-2$ & 1.00 & & & & 1.00 & & & \\
\hline$\geq 3$ & 1.04 & 0.49 & 2.19 & 0.93 & 2.03 & 0.85 & 4.84 & 0.11 \\
\hline \multicolumn{9}{|l|}{ Polypharmacy } \\
\hline$<5$ & 1.00 & & & & 1.00 & & & \\
\hline$\geq 5$ & 0.59 & 0.31 & 1.15 & 0.12 & 1.44 & 0.84 & 2.47 & 0.19 \\
\hline \multicolumn{9}{|l|}{ GDS-5 score } \\
\hline$<2$ & 1.00 & & & & 1.00 & & & \\
\hline$\geq 2$ & 1.63 & 0.82 & 3.25 & 0.17 & 1.71 & 0.86 & 3.41 & 0.13 \\
\hline \multicolumn{9}{|l|}{$\begin{array}{l}\text { Protein intake } \\
\text { (g/kg/day) }\end{array}$} \\
\hline$<0.8$ & 2.22 & 0.68 & 7.27 & 0.19 & 0.35 & 0.08 & 1.44 & 0.15 \\
\hline $0.8-1$ & 1.01 & 0.28 & 3.65 & 0.99 & 0.27 & 0.06 & 1.14 & 0.07 \\
\hline$\geq 1$ & 1.00 & & & & 1.00 & & & \\
\hline
\end{tabular}

GDS-5, Geriatric depression scale-5. ${ }^{\#}$ Adjusted R squared $=0.176 .{ }^{*}$ Adjusted R squared $=0.17$. 


\section{Discussion}

This is one the first studies to investigate the association between health literacy and frailty in community-dwelling healthy elderly. Our results showed that low health literacy was associated with a non-robust status including pre-frailty and frailty. Therefore, aside from nutrition and exercise habits, health literacy seems to be another modifiable factor for frailty interventions.

The relationship between health literacy and frailty may potentially be explained by behavior change theory. Although frailty interventions including nutritional support and exercise programs have been shown to be effective [21], participation and adherence to intervention programs play key roles in improving outcomes. Owing to the current low adherence rate to interventions, it is necessary to emphasize the importance of maintaining behavioral changes [22]. Health literacy has been shown to result in positive reinforcement in seeking information, changing attitudes, and behavioral changes [9], and it has been broadly investigated and associated with the long-term outcomes of smoking cessation, diabetes control, and medication adherence [23-25]. However, the relationship between health literacy and frailty has seldomly been reported. Our findings show that health literacy was an independent predictor of pre-frailty and frailty after controlling for education level, which is consistent with another cross-sectional study [12]. By increasing motivation and encouraging autonomy, health literacy has been shown to improve long-term adherence to frailty management strategies [26].

Of note, our results suggest that sufficient health literacy may be not be sufficient to prevent frailty in the elderly. In the subgroup analysis of physical activity, lower health literacy in the participants with insufficient physical activity was associated with an increased risk of pre-frailty and frailty. Although the trend was not significant in the sufficiently/highly physically active group, sufficient health literacy still remained a protective factor for pre-frailty and frailty. Therefore, the implementation of health literacy promotion appears to be an important issue.

From the viewpoint of stakeholders and policy makers, it has been proposed that frailty management should involve increasing awareness and understanding of frailty among the general public $[27,28]$. In addition, an integrated individualized knowledge translation strategy has been suggested as the initial step to implement frailty prevention and management in the community [28]. Considering the perception gap in community-dwelling elderly, promoting health literacy may both improve knowledge and also reinforce a positive attitude and adherence to interventional approaches. In a weight reduction intervention program, improving health literacy was demonstrated to have a positive influence on dietary intake behavior, but not on physical activity habits [29]. Therefore, future intervention studies including health literacy promotion are warranted to investigate the effects on elderly subjects with different levels of health literacy.

Several health literacy measures such as the Rapid Estimate of Adult Literacy in Medicine (REALM) [30], the Test of Functional Health Literacy in Adults (TOFHLA) [31], and the Newest Vital Sign (NVS) [32] have been validated for busy primary care settings. However, these performance-based measures, which are highly time-consuming and examiner-dependent, may be not appropriate for the community settings. On the other hand, HLS-EU-Q which is subjective health-decision based has been concurrently validated with TOFHLA in a population-based sample [33]. To promote frailty prevention for the aging population in the community, HLS-EU-Q seems to be more applicable and appropriate.

There are several limitations to this study. This was a cross-sectional study, and thus causal relationships could not be determined. In addition, owing to the convenience sampling method, the proportion of frailty was lower in our study (2.99\%) than in another cohort study conducted in elderly community-dwelling Taiwanese [34]. However, the incidence of pre-frailty was similar in both studies. The results implied that our target population was relatively less frail and had greater access to health services. In other words, excluding the physically disabled and individuals with impaired activities of daily life may have underestimated the frailty status in the community. Moreover, participants with advanced old age or terminal illness were not recruited in the present study. This may mean that our findings cannot be applied to the whole elderly population. Despite these limitations, due to the complexity and difficulty in eradicating frailty, promotion of frailty prevention 
is of importance and clinical relevance and worth intervening as early as possible. Health literacy can modify the process of understanding and self-awareness of disease and might be a facilitator to improve the effects of frailty intervention. Therefore, our study has potential to trigger more studies to explore the influence of health literacy on frailty management for the aging population.

\section{Conclusions}

This study shows that health literacy is associated with frailty regardless of age, gender, socio-economic status, and education level in a population of community-dwelling older adults from Taiwan. To enhance recognition of frailty and improve frailty management, health literacy should be considered as a modifiable factor for frailty intervention strategies

Author Contributions: Conceptualization, C.H.H., K.-M.K., and Y.-C.L. (Yu-Cheng Lai); Methodology, C.H.H., K.-M.K., and Y.-C.L. (Yu-Cheng Lai); Formal Analysis, C.H.H., K.-M.K., and Y.-C.L. (Yu-Cheng Lai); Investigation, Y.-C.L. (Yi Chen Lee) and X.T.T.; Data Curation, C.H.H., K.-M.K., and Y.-C.L. (Yu-Cheng Lai); Writing-Original Draft Preparation, C.H.H.; Writing—Review \& Editing, C.H.H., K.-M.K., Y.-C.L. (Yu-Cheng Lai), and M.K.; Supervision, C.H.H.; Project Administration, C.H.H.; Funding Acquisition, C.H.H. All authors approved the manuscript and agree to be accountable for all aspects of the work.

Funding: This study was supported by a grant to Chi Hsien Huang from EDA Hospital Project EMRP-105-79. No financial disclosures were reported by all authors. The funding body had no roles in the study design, data collection, data analysis, and interpretation, or the report writing.

Acknowledgments: We want to show gratitude to the staff of the Department of Community Medicine (Lo, Chan-Yu) for carrying out the data collection. We would also like appreciate community volunteers for their collaborative contribution on health promotion in Southern Taiwan. Last but not least, we are grateful to Beatriz Arakawa Martins for final English editing and correction.

Conflicts of Interest: The authors declared no potential conflicts of interest with respect to the research, authorship, and/or publication of this article.

\section{References}

1. Dent, E.; Lien, C.; Lim, W.S.; Wong, W.C.; Wong, C.H.; Ng, T.P.; Woo, J.; Dong, B.; de la Vega, S.; Hua Poi, P.J.; et al. The Asia-Pacific Clinical Practice Guidelines for the Management of Frailty. J. Am. Med. Dir. Assoc. 2017, 18, 564-575. [CrossRef] [PubMed]

2. Bandeen-Roche, K.; Xue, Q.L.; Ferrucci, L.; Walston, J.; Guralnik, J.M.; Guralnik, P.; Zeger, S.L.; Fried, L.P. Phenotype of frailty: Characterization in the women's health and aging studies. J. Gerontol. A Biol. Sci. Med. Sci. 2006, 61, 262-266. [CrossRef] [PubMed]

3. Crome, P.; Lally, F. Frailty: Joining the giants. CMAJ 2011, 183, 889-890. [CrossRef] [PubMed]

4. Ensrud, K.E.; Ewing, S.K.; Cawthon, P.M.; Fink, H.A.; Taylor, B.C.; Cauley, J.A.; Dam, T.T.; Marshall, M.L.; Orwoll, E.S.; Cummings, S.R.; et al. A comparison of frailty indexes for the prediction of falls, disability, fractures, and mortality in older men. J. Am. Geriatr. Soc. 2009, 57, 492-498. [CrossRef] [PubMed]

5. Fried, L.P.; Tangen, C.M.; Walston, J.; Newman, A.B.; Hirsch, C.; Gottdiener, J.; Seeman, T.; Tracy, R.; Kop, W.J.; Burke, G.; et al. Frailty in Older Adults: Evidence for a Phenotype. J. Gerontol. A Biol. Sci. Med. Sci. 2001, 56, M146-M157. [CrossRef] [PubMed]

6. Lin, S.Y.; Lee, W.J.; Chou, M.Y.; Peng, L.N.; Chiou, S.T.; Chen, L.K. Frailty Index Predicts All-Cause Mortality for Middle-Aged and Older Taiwanese: Implications for Active-Aging Programs. PLoS ONE 2016, 11, e0161456. [CrossRef] [PubMed]

7. Inzitari, M.; Ruiz, D.; Martos, J.; Santaeugenia, S. “Move on Against Frailty”: Time to Raise Awareness about Frailty and Prevention of Disability in the Community. J Frailty Aging 2016, 5, 201-203. [CrossRef] [PubMed]

8. Orlando Frailty Conference, G. Raising Awareness on the Urgent Need to Implement Frailty into Clinical Practice. J. Frailty Aging 2013, 2, 121-124.

9. Sorensen, K.; Van den Broucke, S.; Fullam, J.; Doyle, G.; Pelikan, J.; Slonska, Z.; Brand, H.; (HLS-EU) Consortium Health Literacy Project European. Health literacy and public health: A systematic review and integration of definitions and models. BMC Public Health 2012, 12, 80. [CrossRef] [PubMed] 
10. Aaby, A.; Friis, K.; Christensen, B.; Rowlands, G.; Maindal, H.T. Health literacy is associated with health behaviour and self-reported health: A large population-based study in individuals with cardiovascular disease. Eur. J. Prev. Cardiol. 2017, 24, 1880-1888. [CrossRef] [PubMed]

11. Friis, K.; Vind, B.D.; Simmons, R.K.; Maindal, H.T. The Relationship between Health Literacy and Health Behaviour in People with Diabetes: A Danish Population-Based Study. J. Diabetes Res. 2016, 7823130. [CrossRef] [PubMed]

12. Shirooka, H.; Nishiguchi, S.; Fukutani, N.; Adachi, D.; Tashiro, Y.; Hotta, T.; Morino, S.; Nozaki, Y.; Hirata, H.; Yamaguchi, M.; et al. Association between comprehensive health literacy and frailty level in community-dwelling older adults: A cross-sectional study in Japan. Geriatr. Gerontol. Int. 2016. [CrossRef] [PubMed]

13. Liou, Y.M.; Jwo, C.J.; Yao, K.G.; Chiang, L.C.; Huang, L.H. Selection of appropriate Chinese terms to represent intensity and types of physical activity terms for use in the Taiwan version of IPAQ. J. Nurs. Res. 2008, 16, 252-263. [CrossRef] [PubMed]

14. Hoyl, M.T.; Alessi, C.A.; Harker, J.O.; Josephson, K.R.; Pietruszka, F.M.; Koelfgen, M.; Mervis, J.R.; Fitten, L.J.; Rubenstein, L.Z. Development and testing of a five-item version of the Geriatric Depression Scale. J. Am. Geriatr. Soc. 1999, 47, 873-878. [CrossRef] [PubMed]

15. Duong, V.T.; Lin, I.F.; Sorensen, K.; Pelikan, J.M.; Van Den Broucke, S.; Lin, Y.C.; Chang, P.W. Health Literacy in Taiwan: A Population-Based Study. Asia Pac. J. Public Health 2015, 27, 871-880. [CrossRef] [PubMed]

16. Sorensen, K.; Van den Broucke, S.; Pelikan, J.M.; Fullam, J.; Doyle, G.; Slonska, Z.; Kondilis, B.; Stoffels, V.; Osborne, R.H.; Brand, H.; et al. Measuring health literacy in populations: Illuminating the design and development process of the European Health Literacy Survey Questionnaire (HLS-EU-Q). BMC Public Health 2013, 13, 948. [CrossRef] [PubMed]

17. HLS-Consortium. Comparative Report of Health Literacy in Eight EU Member States. The European Health Literacy Survey HLS-EU, 2012. Available online: http://www.health-leteracy.eu (accessed on 25 November 2018).

18. Pelikan, J.M.; Röthlin, F.; Ganahl, K. Measuring Comprehensive Health Literacy in General Populations: Validation of Instrument, Indices and Scales of the HLS-EU Study. In Proceedings of the 6th Annual Health Literacy Research Conference, Bethesda, ML, USA, 3-4 November 2014.

19. Chan, D.C.; Tsou, H.H.; Chen, C.Y.; Chen, C.Y. Validation of the Chinese-Canadian study of health and aging clinical frailty scale (CSHA-CFS) telephone version. Arch Gerontol. Geriatr. 2010, 50, e74-e80. [CrossRef] [PubMed]

20. Orme, J.G.; Reis, J.; Herz, E.J. Factorial and discriminant validity of the center for epidemiological studies depression (CES-D) scale. J. Clin. Psychol. 1986, 42, 28-33. [CrossRef]

21. Puts, M.T.E.; Toubasi, S.; Andrew, M.K.; Ashe, M.C.; Ploeg, J.; Atkinson, E.; Ayala, A.P.; Roy, A.; Monforte, M.R.; Bergman, H.; et al. Interventions to prevent or reduce the level of frailty in community-dwelling older adults: A scoping review of the literature and international policies. Age Ageing 2017, 46, 383-392. [CrossRef] [PubMed]

22. Fairhall, N.; Sherrington, C.; Cameron, I.D.; Kurrle, S.E.; Lord, S.R.; Lockwood, K.; Herbert, R.D. A multifactorial intervention for frail older people is more than twice as effective among those who are compliant: Complier average causal effect analysis of a randomised trial. J. Physiother. 2017, 63, 40-44. [CrossRef] [PubMed]

23. Bailey, S.C.; Brega, A.G.; Crutchfield, T.M.; Elasy, T.; Herr, H.; Kaphingst, K.; Karter, A.J.; Moreland-Russell, S.; Osborn, C.Y.; Pignone, M.; et al. Update on health literacy and diabetes. Diabetes Educ. 2014, 40, 581-604. [CrossRef] [PubMed]

24. Nelson, L.A.; Wallston, K.A.; Kripalani, S.; LeStourgeon, L.M.; Williamson, S.E.; Mayberry, L.S. Assessing barriers to diabetes medication adherence using the Information-Motivation-Behavioral skills model. Diabetes Res. Clin. Pract. 2018, 142, 374-384. [CrossRef] [PubMed]

25. Stewart, D.W.; Adams, C.E.; Cano, M.A.; Correa-Fernández, V.; Li, Y.; Waters, A.J.; Wetter, D.W.; Vidrine, J.I. Associations between health literacy and established predictors of smoking cessation. Am. J. Public Health 2013, 103, e43-e49. [CrossRef] [PubMed]

26. Juul, L.; Rowlands, G.; Maindal, H.T. Relationships between health literacy, motivation and diet and physical activity in people with type 2 diabetes participating in peer-led support groups. Prim. Care Diabetes 2018, 12, 331-337. [CrossRef] [PubMed] 
27. Archibald, M.M.; Ambagtsheer, R.; Beilby, J.; Chehade, M.J.; Gill, T.K.; Visvanathan, R.; Kitson, A.L. Perspectives of Frailty and Frailty Screening: Protocol for a Collaborative Knowledge Translation Approach and Qualitative Study of Stakeholder Understandings and Experiences. BMC Geriatr. 2017, 17, 87. [CrossRef] [PubMed]

28. Gwyther, H.; Shaw, R.; Jaime Dauden, E.A. Understanding frailty: A qualitative study of European healthcare policy-makers' approaches to frailty screening and management. BMJ Open 2018, 8, e018653. [CrossRef] [PubMed]

29. Cheong, S.M.; Mohamad Nor, N.S.; Ahmad, M.H.; Manickam, M.; Ambak, R.; Ambak, S.N.; Ambak, T. Improvement of health literacy and intervention measurements among low socio-economic status women: Findings from the MyBFF@home study. BMC Womens Health 2018, 18, 99. [CrossRef] [PubMed]

30. Davis, T.C.; Long, S.W.; Jackson, R.H. Rapid estimate of adult literacy in medicine: A shortened screening instrument. Fam. Med. 1993, 25, 391-395. [PubMed]

31. Parker, R.M.; Baker, D.W.; Williams, M.V.; Nurss, J.R. The test of functional health literacy in adults: A new instrument for measuring patients' literacy skills. J. Gen. Intern. Med. 1995, 10, 537-541. [CrossRef] [PubMed]

32. Weiss, B.D.; Mays, M.Z.; Martz, W.; Castro, K.M.; DeWalt, D.A.; Pignone, M.P.; Mockbee, J.; Hale, F.A. Quick assessment of literacy in primary care: The newest vital sign. Ann. Fam. Med. 2005, 3, 514-522. [CrossRef] [PubMed]

33. Toci, E.; Burazeri, G.; Sorensen, K.; Kamberi, H.; Brand, H. Concurrent validation of two key health literacy instruments in a South Eastern European population. Eur. J. Public Health 2015, 25, 482-486. [CrossRef] [PubMed]

34. Liu, L.K.; Lee, W.J.; Chen, L.Y.; Hwang, A.C.; Lin, M.H.; Peng, L.N.; Chen, L.K. Association between Frailty, Osteoporosis, Falls and Hip Fractures among Community-Dwelling People Aged 50 Years and Older in Taiwan: Results from I-Lan Longitudinal Aging Study. PLoS ONE 2015, 10, e0136968. [CrossRef] [PubMed]

(C) 2018 by the authors. Licensee MDPI, Basel, Switzerland. This article is an open access article distributed under the terms and conditions of the Creative Commons Attribution (CC BY) license (http:/ / creativecommons.org/licenses/by/4.0/). 